\begin{tabular}{|c|c|}
\hline $\begin{array}{r}\text { ARTICLES } \\
\text { ARTICULOS }\end{array}$ & $\begin{array}{l}\text { INVESTIGACION: } \\
\text { comprensión de la teoría del periodismo } \\
\text { (contribuciones colombianas) } 1\end{array}$ \\
\hline $\begin{array}{r}\text { Copyright @ } 2013 \\
\text { SBPjor / Associação } \\
\text { Brasileira de } \\
\text { Pesquisadores em } \\
\text { Jornalismo }\end{array}$ & $\begin{array}{l}\text { RAUL HERNANDO OSÓRIO VARGAS } \\
\text { Universidad de Antioquia (Colombia) }\end{array}$ \\
\hline
\end{tabular}

RESUMEN - La historia del periodismo es un espacio de contactos y préstamos. Por ese motivo, la investigación en periodismo tiene una agenda amplia y compleja que abarca, entre otros, temas como: el proceso de producción periodística y su construcción del lenguaje a través de diferentes formas, estructuras y tiempos; metodología, epistemología y la investigación cualitativa en periodismo; historia de los periodistas y del periodismo. Es decir, las relaciones múltiples del periodismo con la cultura, la memoria, las teorías sociales y el discurso, campos que han sido abordados e investigados en Iberoamérica. Sin embargo, la pregunta sobre nuestro etos profesional continúa siendo el eje transversal de la investigación, no solo en el continente americano, sino en el mundo. Es fundamental en nuestra existencia y en el análisis profundo de ese conjunto de características y modos de comportamiento que componen el carácter y la identidad de nuestra profesión.

Palabras clave: Historia y teoría del periodismo. Epistemología. Reportaje.

\title{
PESQUISA: compreensão da teoria do jornalismo (contribuições colombianas)
}

RESUMO - A história do jornalismo é um campo de contatos e empréstimos. Por esse motivo, a pesquisa em jornalismo tem e traz uma agenda ampla e complexa, como: o processo de produção jornalística, sua construção da linguagem por meio de diferentes formas, estruturas e tempos. A metodologia, epistemologia e pesquisa qualitativa em jornalismo; a história dos jornalistas e do jornalismo; ou seja, as relações múltiplas do jornalismo com a cultura, a memória, as teorias sociais e o discurso; campos que têm sido abordados e pesquisados na Ibero-América. Mas a pergunta sobre nosso Ethos profissional é o que continua a ser o eixo transversal da pesquisa, não diria apenas no continente americano, mas no mundo. Ela é fundamental em nossa existência e na análise profunda sobre esse conjunto de características e modos, de comportamentos, que compõem o caráter e a identidade da nossa profissão.

Palavras-chave: História e teoria do jornalismo. Epistemologia. Reportagem.

\section{RESEARCH: understanding the theory of journalism (colombian contributions)}

\begin{abstract}
The history of journalism is a field of contacts and loans. For this reason, research in journalism has and brings a broad and complex agenda, including: the process of news production, its language construction through different forms, structures and tenses. The methodology, epistemology and qualitative research in journalism; the history of journalists and journalism; i.e., the multiple relationships between journalism and culture, memory, social theories and discourse; fields that have been studied and researched in Latin America. But the question on our professional Ethos is what remains the transverse axis of the research, not only in the American continent, but in the world. It is fundamental to our existence and to the depth analysis of this set of features and modes, behaviors, that make up the character and identity of our profession.

Keywords: History and theory of journalism. Epistemology. Report.
\end{abstract}


INVESTIGACIÓN: COMPRENSIÓN DE LA TEORÍA DEL PERIODISMO

"El uno es dos, siempre dos.

Unidad y dualidad son, como el espacio y el tiempo,

inseparables y distintas.

El mismo razonamiento es válido para el uno y el otro, que no son sino variantes de la dualidad original .

Del mismo modo que el uno está en el dos,

el otro está en el ser".2

Octavio Paz

\section{PRESENTACIÓN}

Investigar en sus diversas dimensiones y profundidades el etos, ha sido el punto de partida, que como hilo conductor nos ha permitido estudiar nuestra personalidad histórica, muy vinculada a la teoría de la vida periodística, de su ética y estética, en sus diferentes fases como fenómeno social, cultural, técnico, profesional y científico.

Recordemos que etos también es la raíz de ética. La ética, a su vez, está íntimamente vinculada a la estética, que reflexiona sobre las formas y modos de narración de los acontecimientos, tema que también ha ocupado a estudiosos del periodismo en América Latina. Pensemos, por ejemplo, en los estudios realizados sobre la obra periodística de José Martí, en especial la labor que desempeñó desde los Estados Unidos como corresponsal, en el período de 1880 a 1892, las Ilamadas Escenas Norteamericanas. Estos artículos de Martí constituyen el más penetrante análisis hecho por un escritor y pensador de lengua española.

Sin embargo, Martí dejó clara una novedosa propuesta: el artículo de prensa debía asumir la función pública de lo literario. Susana Rotker, periodista e investigadora venezolana, en su trabajo Fundación de una Escritura, premio Casa de las Américas de 1991, afirma: "La transformación de la escritura -y por ende, de los modos de percepción de la realidad- fue de tal importancia que el periodismo se convirtió, así, en el vehículo de los primeros textos verdaderamente propios en América Latina" (ROTKER, 1992, p. 9).

En la investigación mencionada Susana Rotker, demuestra que más de la mitad de la obra escrita por José Martí y dos tercios de la producida por Rubén Darío, se componen de textos periodísticos. El crítico uruguayo Ángel Rama, en su investigación Rubén Darío y el Modernismo, encontró que "la búsqueda de lo insólito, los acercamientos 
bruscos de elementos disímiles, la renovación permanente, las audacias temáticas, el registro de los matices, la mezcla de las sensaciones" (RAMA, 1970, p.15) fueron la esencia de las transformaciones sociales de finales del siglo XIX y de la experiencia periodística interpretada como la incipiente profesionalización del escritor. El nacimiento del periodismo literario hispanoamericano, al cumplirse en manos de intelectuales excepcionales, mostró el camino de la dignificación de la actividad periodística.

El periodismo como construcción y práctica social es el lugar, o ámbito intelectual, desde donde se conforma su teoría. Cabe destacar que desde 1638 ya se producía un trabajo periodístico en América Latina, hecho confirmado por la investigación sobre El periodismo narrativo en Colombia (1638-2000), realizada por el estudioso y periodista Juan José Hoyos y en la cual afirma:

\begin{abstract}
En medio del olor a polvo y humedad de la gran colección de periódicos y revistas colombianos de los siglos XIX y XX de la Biblioteca de la Universidad de Antioquia, y en los libros de su Colección Patrimonial, aprendí casi todo lo que sé de nuestro pasado y, por lo mismo, de nuestro presente. También del arte de contar. Estas son mis raíces. Allí nació este libro. Su propósito es brindar a los lectores un panorama de la historia del periodismo narrativo en Colombia, de los autores que la construyeron y de la evolución de su estilo (HOYOS, 2009, p. xxi).
\end{abstract}

Por su parte, la investigadora Maryluz Vallejo Mejía, en su obra A plomo herido, estudia la trayectoria del periodismo en Colombia de 1880 a 1980. En dicha investigación reconstruye la historia política y sociocultural del periodismo escrito en Colombia, y su análisis cruza las diversas voces de los propios periodistas, testigos de su época.

Pero no son las empresas, con sus vaivenes, los protagonistas de
esta historia, sino los periodistas. De ellos tomamos sus ideas sobre
el periodismo, producto de su formación intelectual, ilustramos
sus maneras de escribir y de interpretar el país, y seguimos su
trayectoria. Con las voces de los colegas y contemporáneos
también reconstruimos sus perfiles para mostrarlos en su grandeza
y en su pequeñez, con sus aciertos y sus contradicciones, sobre
todo cuando formaron parte del juego de poderes. De ese paso,
se desmitifican unas cuantas figuras siempre incuestionables y
veneradas en la historiografía; se caen del cielo algunos santos,
beatos y canonizados. Son estos retratos sin afeites ni adulaciones,
a menudo irreverentes, los que revelan la condición humana, esa
que da sentido a la obra periodística tan cercana a la literatura. Y
lo que se demuestra hasta la saciedad es que el periodismo en
Colombia, quizá más que en ningún otro país latinoamericano,
está indisolublemente unido a la literatura (VALLEJO, 2006, p. 12).

A su vez, Andrés Vergara Aguirre en la investigación de su tesis 
doctoral titulada Historia del arrabal bogotano en la prensa, 1924-1946. Representaciones de la ciudad y sus infames en las crónicas de Ximénez y Osorio Lizarazo ${ }^{3}$, concluye que los neofolletinistas son una categoría de reporteros en los que se fusionan las técnicas propias de la novela de folletín, algunas tendencias del periodismo sensacionalista y los nuevos géneros periodísticos narrativos, que les permiten producir relatos que resultan atractivos también para los nuevos lectores populares de una Bogotá en proceso de transición hacia la gran urbe, entre 1925 y 1945. Esto, ligado al proceso de industrialización que vivió la ciudad, facilitó el crecimiento acelerado del mercado para las revistas y periódicos en los que escribían los reporteros, entre los que se destacan Ximénez y Osorio Lizarazo: "Por todo ello, podemos afirmar que los neofolletinistas están en el vértice de los procesos de modernización e industrialización de la prensa bogotana"4 (VERGARA, 2012, p. 12).

Uno de los grandes tesoros de la sala de periódicos de la Universidad de Antioquia es la colección Mundo al Día, periódico que nació en 1924 y publicó buena parte del trabajo periodístico del escritor José Antonio Osorio Lizarazo ${ }^{5}$, quien, en distintas etapas, fue el reportero estrella de esta publicación. Osorio Lizarazo tiene una obra muy amplia, pero muchas de sus crónicas están pérdidas en viejos periódicos que hacen difícil su lectura.

Andrés Puerta Molina, en su investigación de maestría sobre el periodismo narrativo de Osorio Lizarazo (PUERTA MOLINA, 2009), recupera parte del trabajo periodístico de Osorio Lizarazo no incluido en los dos libros que recogen sus textos La cara de la miseria (1926) y Novelas y crónicas de J.A Osorio Lizarazo (1978).

En su investigación, Puerta Molina, da a conocer el trabajo de periodismo narrativo de uno de los mejores exponentes que ha tenido Colombia. Para conseguir ese objetivo, hizo un rastreo de periódicos, transcribió y reprodujo los textos, de tal manera que los lectores e investigadores en periodismo tienen acceso a los escritos analizados.

Francisco de Paula Muñoz (Medellín, 1840-1914) es uno de los periodistas y escritores más importantes de Antioquia durante la segunda mitad del siglo XIX y la primera década del siglo XX. Al mismo tiempo es uno de los autores más desconocidos no sólo para las nuevas generaciones de periodistas, sino también para los historiadores y los investigadores que han estudiado el periodismo. En 1874, Muñoz se dedicó durante más de un año a investigar -como periodista- un crimen que había conmovido a la ciudad de Medellín y escribió una narración de 260 páginas "a medida del desarrollo de los sucesos y con toda la 
escrupulosa imparcialidad" (HOYOS, 2002, p. 11) de que era capaz, en momentos en que aún se ignoraba el desenlace de la historia.

Apenas se logró la captura y juzgamiento de los responsables, Muñoz publicó la historia completa de los hechos en un libro titulado: El crimen de aguacatal. El libro empezó a componerse en la Imprenta del Estado de Antioquia en 1874, pero no salió a la luz pública hasta el año siguiente, una vez concluido el juicio de Daniel Escovar y los demás acusados de tomar parte en el crimen.

El libro de Francisco de Paula Muñoz es una muestra temprana y singular del reportaje en Colombia, en un momento en que en los periódicos ni siquiera se usaba esa palabra. Recordemos que el término 'reportaje', según afirma Hobsbawm, es recogido por primera vez en los diccionarios franceses en 1929 y en 1931 por los ingleses: "Se atribuye al periodista comunista checo Erwin Kisch el haber puesto de moda el término en Europa central, en la década de 1920" (HOBSBAWM, 1995, p. 195).

Muñoz se convirtió en uno de los precursores del reportaje en Colombia. Un pionero del reportaje (HOYOS, 2002) es una investigación que estudia el libro de Muñoz en el que se emplean muchas de las técnicas y métodos que, muchos años después, a lo largo del siglo XX, se emplearon en el reportaje moderno.

En 1957, en Argentina, el periodista Rodolfo Walsh publica Operación Masacre, reportaje que tuvo un desarrollo tan expresivo que ha sido considerado el primer libro-reportaje moderno. Sobre este autor, más conocido internacionalmente, existen variadas investigaciones, desde la perspectiva de la teoría del periodismo e, incluso, de la narrativa contemporánea latinoamericana.

Y si fisgamos un poco en la historia, en un sentido panorámico, solo como ejemplo, podemos encontrar lo siguiente: desde los siglos XIII y XIV hasta nuestros días se conservan referencias explicitas de todos los productos informativos elaborados por el periodismo. Además, existe una continuidad lógica en la actividad periodística y una evolución controlable e inteligible. Sin embargo, el periodista es fundamentalmente el "ser humano del Renacimiento", cuando aparecen las gacetas italianas entre los siglos XIV y XVI.

Existen registros que muestran que uno de los primeros periódicos que apareció en América Latina fue la Hoja de México, publicado en el año 1541, en la cual se narraban los sucesos acaecidos durante el terremoto de Guatemala. En el siglo XVII, proliferaron en nuestro continente, especialmente en México y Lima, hojas volantes 
que se publicaban con las noticias de la época. Un siglo más tarde se establecieron periódicos continuos y con secciones diferentes. La Gaceta de México es del año 1722. En 1729, en Guatemala, apareció la Gaceta de Guatemala y en Costa Rica, la Gaceta Mensual. En el Perú se publicaron La Gaceta de Lima en el año 1743 y el Diario de Lima en 1790.

La lista continúa y van apareciendo más países y aumentando el número de periódicos, que son el registro de la vida del periodismo. Contrastar su estudio y análisis con otras fuentes y otras miradas nos permite ver la dimensión de la personalidad histórica de los periodistas de la época y sus variadas problemáticas. El periodismo como objeto de estudio y la importancia de la reflexión teórica sobre su proceso histórico nos llevan a conocer los espacios y tiempos de los periodistas de las diferentes épocas, a fin de valorar adecuadamente el presente. Se trata, pues, de ampliar el alcance de los estudios de periodismo, dotándolos de "un sentido de memoria sobre los medios y sus manejos históricos, así como sobre la evolución del mundo del periodismo" (LÓPEZ, 2005, p. 15).

Pero un mundo pensando en el otro. Desde esa óptica, Juan José García (1997, p. 182), en su investigación sobre La Dimensión Hermenéutica del Periodismo, resultado de consultas y observaciones previas y de su confrontación permanente con la realidad del periodismo colombiano, cuestiona:

¿Puede afirmarse, sin lugar a dudas, que sea tan humano el periodismo como acostumbra creerse, si la relación que plantea no está fundamentalmente en el compromiso con la solidaridad humana, en el respeto a la dignidad de la persona y en el trabajo consecuente por la concreción de los derechos fundamentales? ¿Cómo superar el viejo y erróneo criterio de considerar al otro como objeto, como instrumento, como dato estadístico y muchas veces como nadie?

Y él mismo responde:

Nosotros es una de las palabras clave de nuestra atormentada situación histórica. El otro se nos ha hecho a todos realidad ineludible, y todos hemos adquirido viva conciencia de ello. He aquí una clara y concluyente respuesta al interrogante inicial sobre a quién le sirve el periodismo y por qué es importante la búsqueda de sentido, en el desarrollo del planteamiento hermenéutico (GARCÍA, 1997, p. 183).

Estas reflexiones sobre el periodismo, su práctica y su teoría nos permiten ver las diversas dimensiones de la investigación en el área, sus representaciones y el contexto en que fueron producidas, con una visión amplia e interdisciplinar y desde una perspectiva de historia cultural e intelectual del periodismo.

En esa perspectiva, ¿se imaginan si se realizaran investigaciones en toda América Latina, como la hecha por Juan José Hoyos sobre el 
periodismo narrativo en Colombia? Lo que encontraríamos y la teoría del periodismo que emergería de ese iceberg sería muy valioso para la historia y la epistemología del periodismo. O tal vez, parte de esa investigación ya esté siendo realizada y la desconocemos.

Estos son algunos ejemplos de cómo el periodismo, su construcción de la realidad y su reflexión teórica se han presentado en Hispanoamérica. No obstante, si entramos en contacto con los investigadores en periodismo de cada uno de los países del continente y les preguntamos, encontraremos que en nuestras naciones ha habido y hay muchos pioneros; y que se ha hecho y se hace investigación seria y consistente, que contribuye a la conformación de la epistemología del periodismo. Pero, desafortunadamente, no nos integramos, nos desconocemos. Si nos preguntan en América Latina por Truman Capote u Otto Groth, con seguridad, hasta los más jóvenes estudiantes saben quiénes son. Sin embargo, si les preguntamos a esos mismos estudiantes de periodismo hispanoamericanos, si saben quién es Adelmo Genro Filho, pocos lo sabrán.

No estoy de acuerdo con algunos autores que afirman que cada día se hacen menos investigaciones en periodismo: sin duda alguna, hoy se hace mucho más, con mayor profundidad, profesionalismo intelectual y científico. Lo que necesitamos es una red de investigadores de Iberoamérica para ayudar a descubrir, organizar y sistematizar lo que se ha hecho hasta el momento.

En palabras del escritor Carlos Fuentes (2006, p. 1): "Tenemos la gran suerte de que la personalidad iberoamericana es indígena, africana, mulata, mestiza y, a través de Iberia, mediterránea, griega, latina, árabe, judía, cristiana y laica. Somos, podemos ser, también el microcosmos de la convivencia". Pero debemos tener la capacidad de incorporar esta riqueza a la acción político-social, al mundo educacional, a la vida diaria de todos los países iberoamericanos y, fundamentalmente, a la práctica del periodismo y su reflexión teórica, de aquello que Fuentes (1990) denominó realidad sociocultural de Indo-Afro-Ibero-América.

Con todo, también es importante dar un paso adelante y estudiar la teoría del periodismo como crítica cultural, ya que el periodismo es un hábitat de diversidad y complejidad, que se mueve y vive en el tejido social de nuestras realidades. El periodismo como término, concepto, noción, historia, profesión e investigación se ha ido imponiendo en la sociedad contemporánea, no siendo un concepto cerrado. Por el contrario, es una noción abierta, compleja, multidisciplinar, que se interesa por los acontecimientos y sus conexiones. Es el camino de la pluralidad, con sus métodos e investigaciones sobre los sentidos humanos. 


\section{PERIODISMO Y SUS SENTIDOS DE LAS REALIDADES}

¿Lo que les interesa a los periodistas tiene que ver con las necesidades del continente? En mi opinión, deben ser creadas Agendas de Continente, que lleven los temas de los estudios e investigaciones del periodismo hacia los problemas vivos de Iberoamérica. Y esto está relacionado con temas importantes entre ellos: el papel de los periodistas frente a las violencias, los desafíos de las culturas digitales y cómo los periodistas se están apropiando, o no, de esas nuevas narrativas.

El sector que parece más impactado con este cambio de entorno es el educativo; y aquí periodismo y educación forman una pareja indispensable para educar a las personas para pensar y hacerse ciudadanos. De ese modo, el periodismo cumple un papel esencial al construir con su narrativa de los acontecimientos el ejercicio de la ciudadanía, estando, así, vinculado a los nuevos movimientos sociales, que están transformando desde abajo la vida de la gente y la forma de hacer política. Esto supone también una forma nueva de hacer e investigar en periodismo. Es, por tanto, un gran desafío.

En nuestro campo, debemos comenzar a reconocernos, porque no hay una sola manera de ser periodista. Sin una agenda, ¿Cómo podemos recorrer la investigación del periodismo? Los ejemplos que he presentado son mínimos en comparación con la cantidad de investigaciones realizadas en nuestra área. A partir de esa babel de investigaciones, debemos componer una lista inicial que sea desarrollada por todos los participantes de esa red, lo cual incluye la posibilidad de crear un sitio en Internet, para socializar esos conocimientos y dar mayor visibilidad a nuestra área.

Esta lista inicial sería como una guía para tener más elementos con respecto a dónde estamos y qué hacemos, así como para indicarnos hacia dónde ir y con qué horizontes. Esta guía nos permitirá encontrarnos, realizar balances, diagnósticos y proponer acciones, pues el periodismo y el periodista continúan siendo fundamentales para la democracia en el mundo contemporáneo, en la 'Era de los Líquidos'.

En esa perspectiva, Zigmunt Bauman, en su libro Modernidad Líquida apunta:

En su etapa pesada, el capital estaba tan fijado al suelo como los trabajadores que contrataba. En la actualidad, el capital viaja liviano, apenas con equipaje de mano, que incluye tan solo un portafolio, un teléfono móvil y un ordenador portátil. Puede hacer escala en cualquier punto del camino y en ningún lugar se demora más tiempo del que dura su satisfacción. El trabajo, por otro lado, permanece tan inmovilizado como en el pasado, pero el lugar en que imaginaba estar fijado de una vez por todas ha perdido 
solidez; buscando rocas, las anclas encuentran arenas movedizas. Algunos de los habitantes del mundo están en movimiento; para el resto, es el mundo el que no se queda quieto (BAUMAN, 2001, 70-71). ${ }^{6}$

Ahora todo cambia con una velocidad mayor en ese proceso de hacer periodismo y de educar a los periodistas. ¿Qué saber, qué aprender, qué enseñar, qué hacer? ¿Cómo comprometerse? Estos serían los temas para un Congreso Internacional de Educación y Enseñanza del Periodismo.

Como afirmó Edgar Morin en su propuesta La cabeza bien puesta, "la educación puede ayudar a hacernos mejores, si no más felices, y enseñarnos a asumir la parte prosaica y a vivir la parte poética de nuestras vidas" (MORIN, 2001, p. 11).

Debemos, por tanto, dejar de pensar solo en las fronteras y construir las relaciones y los entrelazamientos que dignifican el periodismo y sus caminos (métodos) de investigación en los laberintos globales, a fin de proyectar los cambios en la cultura híbrida de la que habla Néstor García Canclini (2008).

\section{$\left.\right|_{\text {NOTAS }}$}

1 Este trabajo forma parte del proyecto de investigación: Periodismo y reportaje transcultural, desarrollado en la Universidad de Antioquia - Colombia, Sistema Universitario de Investigación - CODI N. ${ }^{0} 584$, y fue presentado en el $10^{\circ}$ Encuentro Nacional de Investigadores en Periodismo, Curitiba, 9 de noviembre de 2012.

2 PAZ, Octavio. Nosotros: los otros. Prólogo al tomo 10 de las Obras Completas: Ideas y costumbres, II, Usos y símbolos. México: Fondo de cultura económica, 1996.

3 José Joaquín Jiménez (Bogotá, 1916-1946), fue uno de los reporteros más populares de Bogotá en aquella época; aquí se le nombra con el seudónimo que más utilizó, Ximénez. José Antonio Osorio Lizarazo (Bogotá, 1900-1964), quien después sería un prolífico novelista, fue otro de los reporteros más reconocidos en los años veinte en Bogotá.

4 Memorias Primer Congreso de Historia Intelectual de América Latina, Medellín, 12-14 de septiembre de 2012. Mesa: Historia del periodismo y la opinión pública en América Latina. 
5 El trabajo periodístico de Osorio Lizarazo fue muy amplio: redactor de El Sol, Bogotá 1922; redactor en Gil Blas, Bogotá 1923-1924; reportero de Mundo al Día, Bogotá 1924-1929; corresponsal en Centro de América de Cromos, Bogotá 1929; redactor de viaje de El Espectador, Bogotá 1929; redactor (1929-1931) de La Prensa, Barranquilla; fundador y jefe de redacción (1933-1934) de El Heraldo, Barranquilla; director de El Diario Nacional, Bogotá 1935-1936; colaborador de la revista Pan, Bogotá 1937-1939; corresponsal de viaje (1939), redactor (1934-1935, 19391940), colaborador (1941-1946) y corresponsal en Argentina (19461950) del periódico El Tiempo de Bogotá; colaborador de la Revista de la Indias, Bogotá 1942-1946, 1954; colaborador de la Revista de América (El Tiempo), Bogotá 1945-1950; fundador y director del periódico Jornada - por la restauración moral de la República, Bogotá 1944-1945; jefe de redacción de Sábado - Semanario al servicio de la cultura y de democracia en América, Bogotá 1945; colaborador de la revista Economía Colombiana, Bogotá 1955; colaborador de Dinámica Social, Buenos Aires 1950-1956 (Revista del Centro de Estudios Económico Sociales), director de El Caribe, Ciudad Trujillo-República Dominicana 1958-1960.

6 Traducción del autor.

REFERENCIAS

BAUMAN, Zigmunt. Modernidade líquida. Rio de Janeiro: Zahar, 2001.

FUENTES, Carlos. Valiente mundo nuevo: Épica, utopía y mito en la novela hispanoamericana. México: Fondo de Cultura Económica, 1990.

FUENTES, Carlos. No hay discurso sin nuestra voz. VII Foro Iberoamérica. Ciudad de México. 30 Noviembre de 2006. Discurso.

GARCÍA CANCLINI, Néstor. Culturas híbridas: estrategias para entrar y salir de la modernidad. Buenos Aires: Paidós, 2008.

GARCÍA POSADA, Juan José. La dimensión hermenéutica del periodismo. Medellín: Editorial Universidad Pontificia Bolivariana, 1997.

HOBSBAWM, Eric John Ernest. Historia del siglo XX. Barcelona: Crítica, 1995.

HOYOS NARANJO, Juan José. La pasión de contar. El periodismo narrativo en Colombia (1638-2000). Medellín: Hombre Nuevo Editores-Editorial Universidad de Antioquia, 2009.

HOYOS NARANJO, Juan José. Un pionero del reportaje: Francisco de Paula Muñoz y El crimen de Aguacatal. Medellín: Hombre Nuevo Editores-Editorial Universidad de Antioquia, Colección de Periodismo, 2002.

LÓPEZ, Fabio. Presentación del Dossier sobre historia de los medios de comunicación social y del periodismo en Colombia. Revista Historia Crítica, Bogotá, Universidad de los Andes, n. 28, 2005. 
MARTÍ, José. "Escenas norteamericanas". In: Obras Completas. La Habana: Editorial Ciencias Sociales, 1986. Tomos 9-12.

MORIN, Edgar. A cabeça bem-feita: repensar a reforma, reformar o pensamento. Rio de Janeiro: Bertrand Brasil, 2001.

PUERTA MOLINA, Andrés Alexander. Una recuperación del olvido para el escritor de los olvidados: Periodismo narrativo de José Antonio Osorio Lizarazo. Disertación de Maestría. Orientador: Doctor Phil. Edison Neira Palacio. Medellín: Universidad de Antioquia, Facultad de Comunicaciones, 2009.

RAMA, Ángel. Rubén Darío y el modernismo. Caracas: Universidad Central de Venezuela, 1970.

ROTKER, Susana. Fundación de una escritura: Las crónicas de José Martí. La Habana: Ediciones Casa de las Américas, 1992.

VALLEJO MEJíA, Maryluz. A plomo herido: Una crónica del periodismo en Colombia (1880-1980). Bogotá: Editorial Planeta, 2006.

VERGARA AGUIRRE, Andrés. Historia del arrabal bogotano en la prensa, 1924-1946. Representaciones de la ciudad y sus infames en las crónicas de Ximénez y Osorio Lizarazo. Tesis de doctorado en Historia, junio de 2012. Universidad Nacional de Colombia, sede Medellín.

WALSH, Rodolfo J. Operación Masacre: un proceso que no ha sido clausurado. Buenos Aires: Colección Documentos, Ediciones Sigla, 1957.

Raúl Hernando Osorio Vargas Magíster y Doctor
en Comunicación, en el área del Periodismo por la
Escuela de Comunicaciones y Artes de la Universidad
de São Paulo. Profesor Asistente del Programa de
Periodismo de la Facultad de Comunicaciones de la
Universidad de Antioquia - Colombia. Miembro del
Grupo de Estudios Literarios, GEL, clasificación A, de
COLCIENCIAS. E-mail: osoriova@gmail.com

RECIBIDO EN: 04/04/2013 | APROBADO EN: 07/07/2013 
INVESTIGACIÓN: COMPRENSIÓN DE LA TEORÍA DEL PERIODISMO 